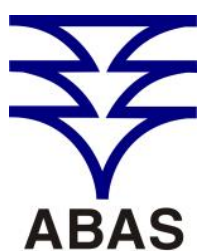

ASSOCIACAO BRASLERRADE
AGUAS SUBTERRANEAS www.abas.org

\section{ESTIMATIVA DA RECARGA DAS ÁGUAS SUBTERÂNEAS DO SISTEMA AQUÍFERO BARREIRAS NA BACIA DO RIO PIRANGI, RN}

\author{
ESTIMATING THE RECHARGE OF GROUNDWATER IN THE \\ BARREIRAS AQUIFER SYSTEM, PIRANGI RIVER BASIN, RN
}

José Geraldo de Melo¹, Rafaela da Silva Alves², Jadsom Gomes da Silva ${ }^{3}$

Artigo recebido em: 09/10/2013 e aceito para publicação em: 18/08/2014.

\begin{abstract}
The groundwater of Barreiras Aquifer System (SAB) in the Pirangi river basin, RN, is a crucial resource for the population's water supply. The groundwater is used, however, without proper planning, through the occupation of important recharge areas and the absence of a sewage system in the urban areas, thus resulting in potential risks for groundwater contamination, potential reduction of exploitable resources, and inefficient use of water. The main objective of this work is to estimate the rate of recharge in the Barreiras Aquifer System, as a way to assess its potential, in order to adopt management measures and to maximize and/or optimize the use of water, with an eye towards the development of the region. The recharge was estimated by applying three different methodologies in order to compare results and choose the most appropriate method. They were "Water-Table Fluctuation Method", Darcy's law applied to the saturated porous medium and the hydrological method, using data from Pirangi River recession. In all cases, urban recharge and water removal through pumping were taken into account. The analysis of the results indicated a recharge of $277,0 \mathrm{~mm} /$ year, which corresponds to an infiltration rate of $18,0 \%$ of the total annual precipitation.
\end{abstract}

Keywords: Recharge of Groundwater. Barreiras Aquifer System. Pirangi River Basin.

Resumo: A água subterrânea do Sistema Aquífero Barreiras (SAB) na bacia hidrográfica do Rio Pirangi, RN, é um recurso de fundamental importância no suprimento hídrico da população. $\mathrm{O}$ uso das águas subterrâneas é feito, entretanto, sem um planejamento adequado, com a ocupação de áreas importantes de recarga e ausência de sistema de esgotamento sanitário nos meios urbanos, resultando, por conseguinte, em riscos potenciais de contaminação das águas subterrâneas, redução em potencial dos recursos explotáveis e ineficiência do uso das águas. O objetivo principal deste trabalho é realizar a estimativa da recarga do sistema aquífero Barreiras, como forma de avaliação das suas potencialidades, visando a adoção de medidas de gestão e a maximização e/ou otimização do uso das águas, tendo em vista o desenvolvimento da região. A recarga foi estimada com a aplicação de três diferentes metodologias tendo em vista a comparação de resultados e a escolha do método mais adequado. Foram aplicados os métodos da variação de carga ("Water-Table Fluctuation Method"), lei de Darcy aplicada ao meio poroso saturado e o método hidrológico utilizando dados de recessão do Rio Pirangi. Em todos os casos, foi considerada a recarga urbana e as retiradas de água por bombeamento. A análise dos resultados indicou uma recarga de 277,0 mm/ano, que corresponde a uma taxa de infiltração de $18 \%$ do total precipitado anualmente.

Palavras-chave: Recarga de águas subterrâneas. Sistema Aquífero Barreiras. Bacia hidrográfica do Rio Pirangi.

\title{
INTRODUÇÃO
}

As águas subterrâneas do Sistema Aquífero Barreiras, na bacia hidrográfica do Rio Pirangi, constituem a principal fonte de suprimento hídrico das populações urbanas e rurais, como também é o manancial utilizado no desenvolvimento de culturas irrigadas e no desenvolvimento industrial.

Para explotar e garantir o uso sustentável de um aquífero, é necessário o conhecimento dos mecanismos e montante da recarga anual no qual o mesmo é submetido. A explotação de um volume maior pode induzir a rebaixamentos indesejáveis e afetar as captações d'água e o próprio aquífero. A recarga nem sempre pode ser explotada integralmente, devendo, por conseguinte, serem observados os aspectos ambientais envolvidos.

A recarga do Sistema Aquífero Barreiras na bacia do Rio Pirangi, foi avaliada com vistas a fornecer subsídios para uma gestão sustentável

\footnotetext{
1 Área de hidrogeologia, Departamento de Geologia, Universidade Federal do Rio Grande do Norte. (jgmelo@ufrnet.br).

2 Área de hidrogeologia, Universidade Federal do Rio Grande do Norte.

(alves.rafaelasilva@yahoo.com.br).

${ }^{3}$ Departamento de Geologia, Universidade Federal do Rio Grande do Norte. (jadsonghfrn@ hotmail.com).
} 
das águas subterrâneas e dentro do possível a maximização do uso das águas para o desenvolvimento da região, com prioridade ao suprimento hídrico urbano.

Em sistemas aquíferos livres, como é o caso do aquífero Barreiras, suas potencialidades estão condicionadas principalmente às infiltrações diretas das águas de chuva, cujo volume efetivamente infiltrado restituem as reservas de águas subterrâneas que se perdem nos escoamentos naturais e explotação por poços e, também, alimentam o fluxo subterrâneo. Por definição, em condições de equilíbrio, sem considerar a explotação por poços ou influência urbana, a infiltração eficaz é equivalente à vazão do fluxo subterrâneo natural e às reservas reguladoras (CASTANY,1976).

Várias técnicas são disponíveis para quantificar recarga, entretanto a escolha de técnicas apropriadas é frequentemente difícil. Técnicas baseadas em dados de águas superficiais e da zona insaturada geralmente fornecem estimativas da recarga potencial, enquanto que aquelas baseadas em dados de águas subterrâneas fornecem estimativas da recarga real (SCANLON et al., 2002). A avaliação dos recursos de águas subterrâneas, geralmente, requer técnicas que forneçam estimativas regionais de recarga (SCANLON et al., 2002), o que é considerado no âmbito deste trabalho.

As dúvidas associadas a cada método corroboram a necessidade de aplicar várias técnicas distintas para aumentar a confiabilidade nas estimativas da recarga.

No interior da bacia do Rio Pirangi está localizado o município de Parnamirim, com área em sua maior parte urbanizada, e, uma pequena parte da Zona Sul da cidade de Natal. Em ambientes urbanos, os mecanismos de recarga natural de aquíferos livres e semi-confinados situados sob seus domínios são modificados e são criados novos mecanismos (FOSTER et al., 1999). Nestes casos, a recarga urbana atribuída a estrutura urbana formada pelo abastecimento de água, saneamento e drenagem devem ser considerados (FOSTER et al., 1999; MELO e QUEIROZ, 2001) .

As tubulações do sistema público de abastecimento estão propensas a vazamentos e estimase que as perdas de água na rede constituem uma importante parcela da recarga urbana. As áreas urbanas, no domínio da bacia do Rio Pirangi, não dispõem de rede de esgotos, o que contribui para o aumento da recarga, mediante a infiltração dos efluentes no terreno. Nestes casos, com riscos de contaminação das águas subterrâneas. A recarga propiciada pelas descargas de efluentes domésti- cos, além de contribuir para a contaminação das águas subterrâneas, apresenta outra influência importante no contexto hidrogeológico, que é a tendência em elevar o nível das águas subterrâneas ou as cargas hidráulicas.

\section{CARACTERIZAÇÃO DA ÁREA}

A bacia hidrográfica do Rio Pirangi está localizada no litoral Oriental do Estado do Rio Grande do Norte, conhecida como região dos vales úmidos. Sobre sua superfície precipita-se uma lâmina d'água média de $1539 \mathrm{~mm} / \mathrm{ano}$ (Série Histórica 1995-2010), segundo informações da Empresa de Pesquisa Agropecuária do Rio Grande do Norte (EMPARN). A bacia é formada pelas sub-bacias dos rios Pitimbu, Taborda e Pium (Figura 1), com superfície total de $436 \mathrm{~km}^{2}$. São rios perenes que se desenvolvem sobre os sedimentos Tercio-Quaternários do Grupo Barreiras, os quais são alimentados por ressurgências de águas subterrâneas do aquífero formado por esses sedimentos que constitui o "Sistema Aquífero Barreiras (SAB)". O Rio Pitimbu, afluente do Rio Pirangi, é o manancial mais estudado do ponto de vista ambiental e hidrológico (DUARTE et al., 2004; BORGES, 2002; KOBAYASHI et al., 2010), haja vista que o mesmo flui para a lagoa de Jiqui, cujas águas são utilizadas no abastecimento da Zona Sul da cidade de Natal. Do ponto de vista geomorfológico, dominam os tabuleiros costeiros com ocorrência de planícies lagunares e elevações dunares que se desenvolvem mais proeminentemente na faixa costeira.

Existe uma ampla relação entre os mananciais superficiais e subterrâneos, sendo o aquífero nitidamente de regime influente nos canais fluviais e lagoas, evidenciado em estudos anteriores (LUCENA et al., 2004).

O Sistema Aquífero Barreiras ocorre em grande parte da bacia hidrográfica do Rio Pirangi, com superfície da ordem de $420 \mathrm{~km}^{2}$ (Figura 1). Trata-se de um pacote de sedimentos formado na sua parte superior de arenitos finos com variação lateral de fácies para arenitos argilosos e argilitos, e, na parte inferior, são dominantemente arenitos médios a grossos com níveis argilosos (Figura 2).

Sobre os sedimentos do Grupo Barreiras, em grande parte da área, ocorrem coberturas de dunas que, embora se apresentem insaturadas, provavelmente têm grande influência no processo de recarga do Sistema Aquífero Barreiras, pela constituição essencialmente arenosa que lhe permite atribuir elevada porosidade efetiva e elevada condutividade hidráulica. 


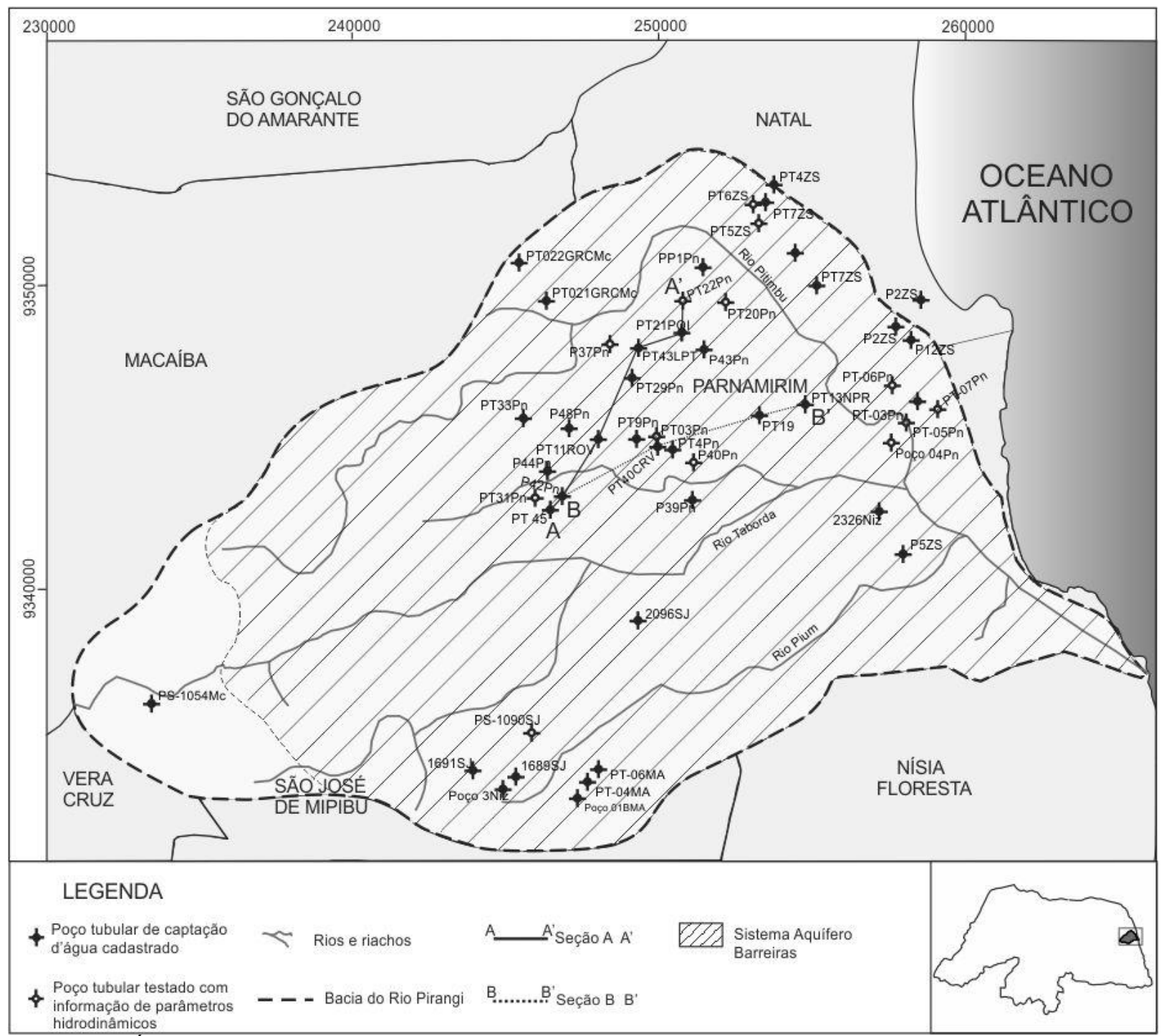

Figura 1 - Área de estudo e distribuição dos poços de captação d'água utilizados nas avaliações hidrogeológicas Figure 1 - Study area and distribution of the wells used in the hydrogeological assessments

A estrutura geológica do terreno tem uma influência marcante sobre as espessuras saturadas do Sistema Aquífero Barreiras, e, por conseguinte sobre as suas potencialidades, o que se reflete na produtividade dos poços, no qual as maiores vazões explotáveis correspondem às maiores espessuras saturadas (BEZERRA et al., 1993; LUCENA E QUEIROZ, 1996; LUCENA et al., 2006).

\section{METODOLOGIA}

A recarga foi estimada com a aplicação de três diferentes metodologias tendo em vista a comparação de resultados e a escolha do método mais adequado. Foram aplicados os métodos da variação de carga ("Water-Table Fluctuation Method"), lei de Darcy aplicada ao meio poroso saturado e o método hidrológico utilizando dados de recessão do Rio Pirangi. Na estimativa da recarga efetiva, com aplicação desses métodos, foi neces- sário estabelecer condições de equilíbrio hidrodinâmico mediante balanço hidrogeológico considerando as retiradas de água por bombeamento e a recarga urbana.

A aplicação dos métodos de avaliação de recarga requereu uma estimativa do volume anual de água bombeado no Sistema Aquífero Barreiras, de suas características dimensionais, hidrodinâmicas e do fluxo subterrâneo, e, de dados hidrológicos da recessão do Rio Pirangi.

\section{Uso e Características dimensionais e hidrodi- nâmicas do Sistema Aquífero Barreiras}

O uso (retiradas por bombeamento) das águas subterrâneas foi avaliado com base nos resultados de um cadastro de poços, considerando as descargas bombeadas e os respectivos regimes de funcionamento. Nesta tarefa, foi utilizado o arquivo de dados da Companhia de Águas e Esgotos do Rio Grande do Norte (CAERN) e da Secre- 
taria de Recursos Hídricos e do Meio Ambiente do Rio Grande do Norte (SEMARH).

As dimensões do Sistema Aquífero Barreiras e de seus parâmetros hidrodinâmicos (condutividade hidráulica, transmissividade e porosi- dade específica) foram avaliados com base na análise dos perfis litológicos e de resultados de testes de bombeamento e de produção, de 31 poços totalmente penetrantes (Tabela 1).

Tabela 1 - Parâmetros hidráulicos do Sistema Aquífero Barreiras na Bacia do Rio Pirangi, RN

Table 1 - Hydraulic parameters of the Barreiras Aquifer System in the Pirangi River

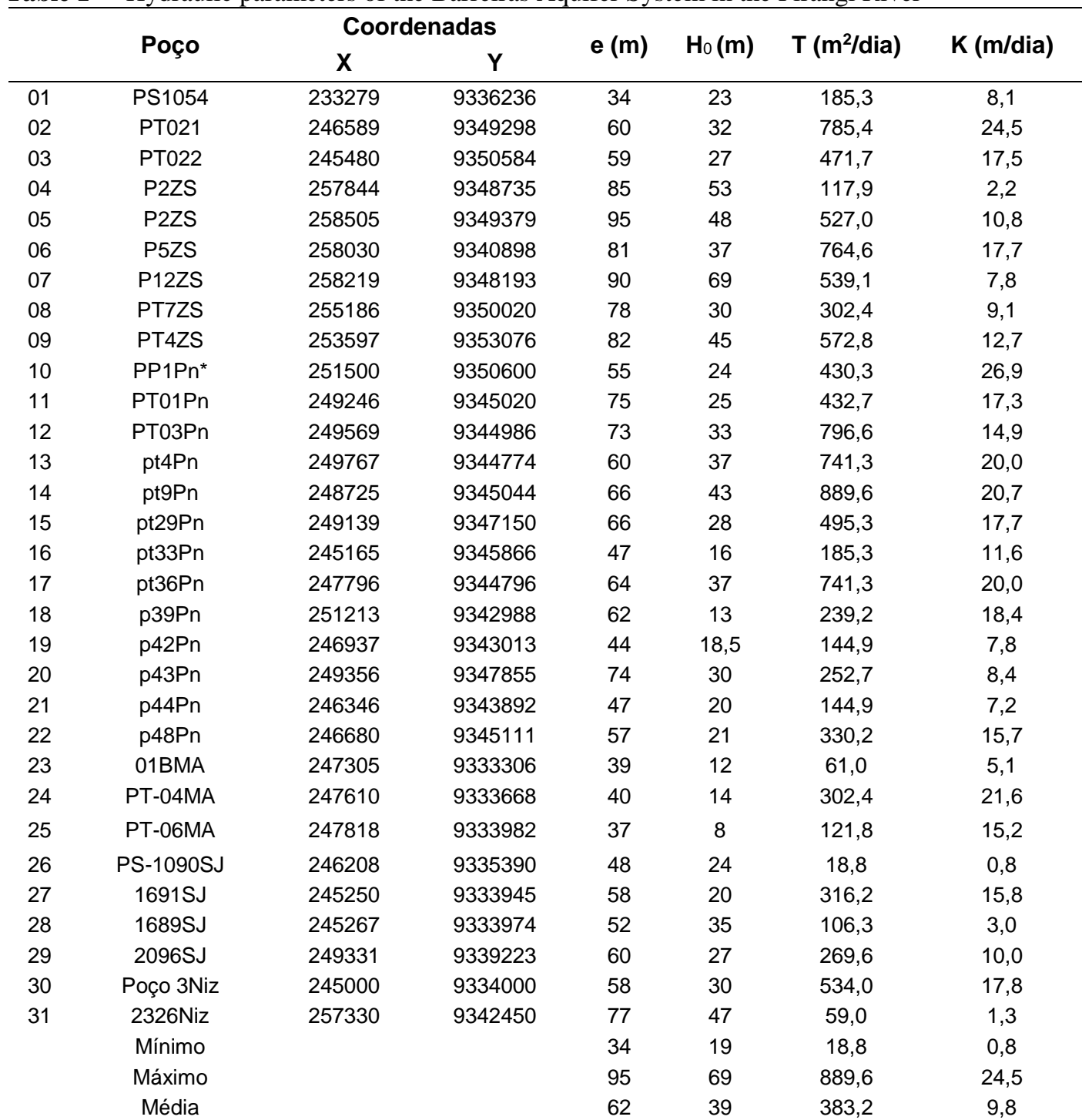

Obs: e:espessura total SAB; $h_{0}$ : espessura saturada SAB; T: transmissividade; K: conditividade hidráulica *: Teste de aquífero.

$\mathrm{Na}$ interpretação dos resultados dos testes foi utilizado o software Aquifer Test Pro, versão 2010 (Schlumberger), com a aplicação do método de Cooper \& Jacob na avaliação da condutividade hidráulica e da transmissividade do aquífero.

No caso da porosidade eficaz ou rendimento específico (Sy), a mesma foi estimada com base na equação de Biecinski (PAZDRO, 1983, in ALVAREZ e NIEDZIELSKI, 1996):

$S y=0,117 \sqrt[7]{K}$
Onde:

$K$ é condutividade hidráulica em $\mathrm{m} /$ dia.

Os resultados dos testes de bombeamento foram fornecidos pela CAERN, SEMARH e Departamento de Geologia da Universidade Federal do Rio Grande do Norte (DG-UFRN).

As características do fluxo subterrâneo são apresentadas com base em mapas potenciométricos já existentes (figuras 3 e 4). Os dados hidrológicos de recessão do Rio Pirangi são resultados de monitoramento mensal obtido por um período de dois anos (Tabela 2). 


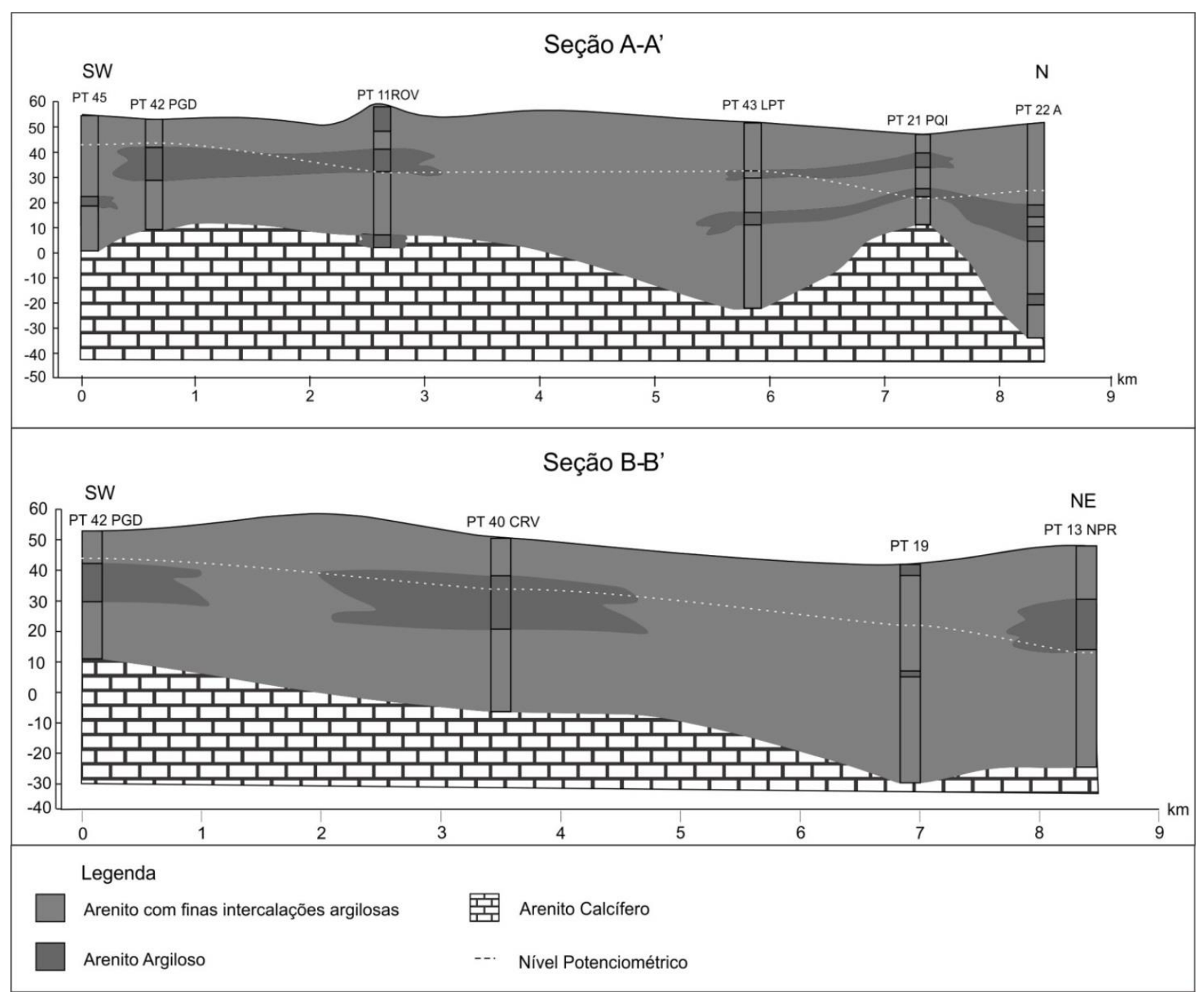

Figura 2 - Seções hidrogeológicas do Sistema Aquífero Barreiras na bacia do Rio Pirangi Figure 2 - Hydrogeological sections of Barreiras Aquifer System in the Pirangi River basin

Tabela 2 - Descargas do Rio Pirangi - baixo curso (período Fevereiro 2000-Dezembro 2001). Valores em $\mathrm{m}^{3} / \mathrm{s}$ Table 2 - Discharge of the Pirangi River - lower course (period February 2000-December 2001). Values in $\mathrm{m}^{3} / \mathrm{s}$

\begin{tabular}{|c|c|c|c|c|c|c|c|c|c|c|c|c|}
\hline & Jan & Fev & Mar & Abr & Mai & Jun & Jul & Ago & Set & Out & Nov & Dez \\
\hline 2000 & & 1,37 & 1,14 & 2,29 & 2,56 & & 6,97 & 7,84 & 7,52 & 5,54 & 5,47 & 4,92 \\
\hline 2001 & & & 6,96 & 4,63 & 3,22 & 3,60 & 3,46 & 3,07 & 2,50 & 2,56 & 2,10 & 2,31 \\
\hline
\end{tabular}

(LUCENA et al., 2006). 


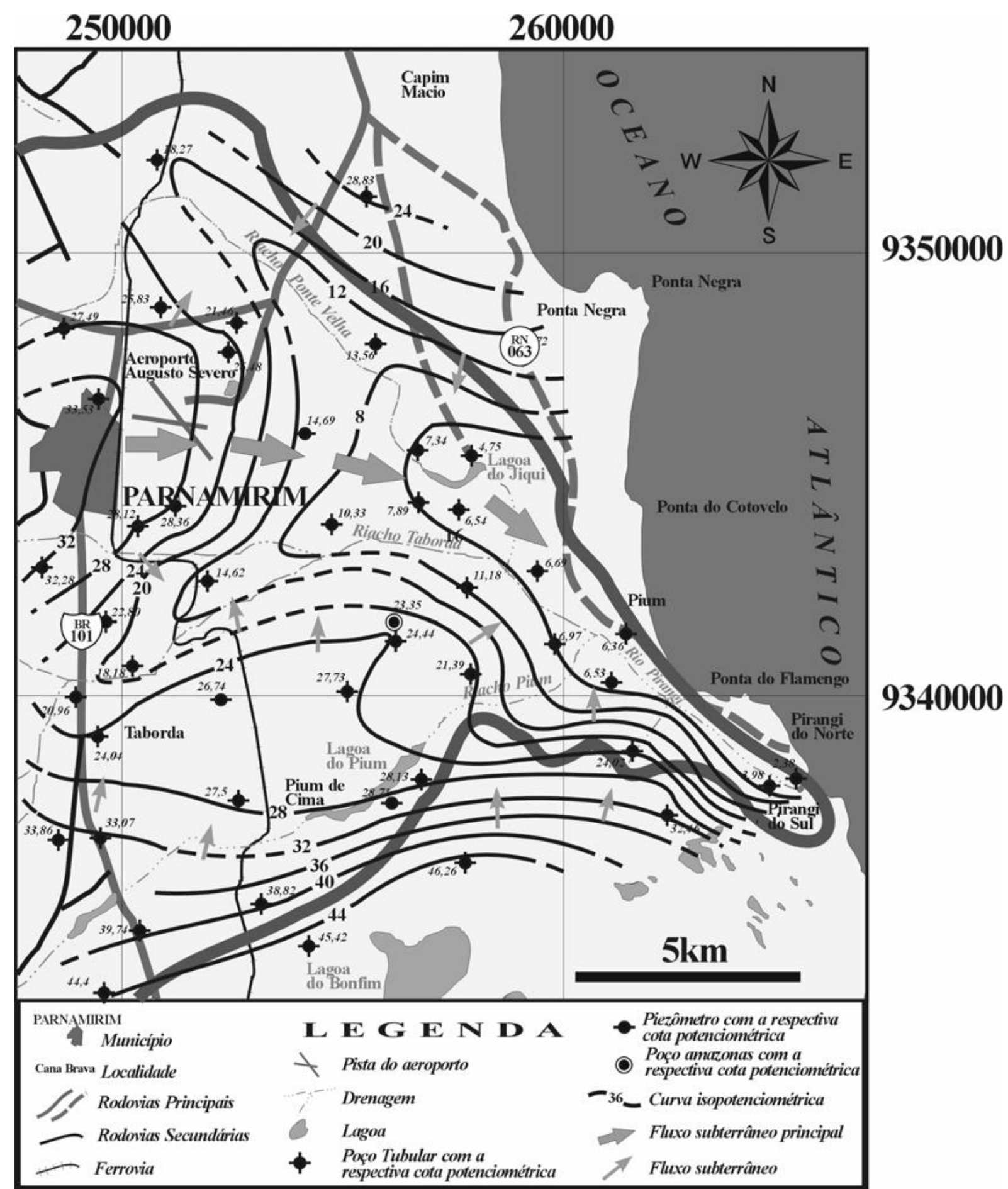

Figura 3 - Mapa potenciométrico do aquífero Barreiras na bacia do Rio Pirangi referente ao período chuvoso Agosto 2000 (LUCENA et al., 2004)

Figure 3 - Potentiometric map of the Barreiras Aquifer System in the Pirangi River basin reffering rainy period August 2000 (LUCENA et al., 2004) 


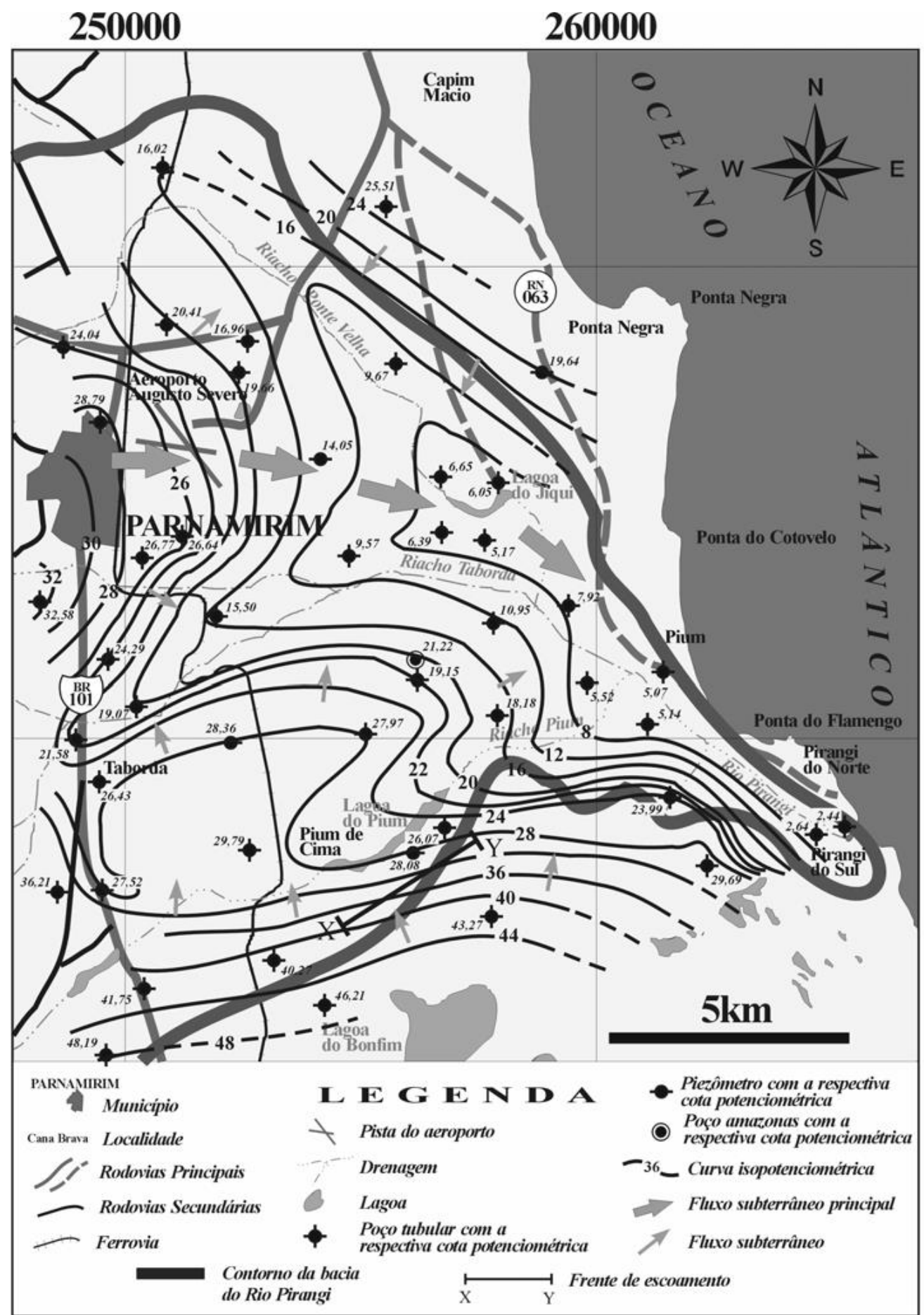

\section{0}

9340000

Figura 4 - Mapa potenciométrico do aquífero Barreiras na bacia do Rio Pirangi referente ao período seco Março 2001 ( LUCENA et al., 2004)

Figure 4 - Potentiometric map of the Barreiras aquifer system in the Pirangi river basin referring dry season March 2001 (LUCENA et al., 2004)

\section{Recarga}

Recarga urbana te equação:

A recarga urbana foi avaliada pela seguin-

$\mathbf{R}_{\mathrm{u}}=\mathbf{q}_{\mathrm{e}}+\mathbf{q}_{\mathrm{v}}$

Onde: $\mathrm{mm} / \mathrm{ano}$

$q_{\mathrm{e}}$ é a descarga dos esgotos em $\mathrm{m}^{3} / \mathrm{ano}$ ou

$q_{\mathrm{v}}$ são as perdas de água na rede em $\mathrm{m}^{3} / \mathrm{ano}$ ou $\mathrm{mm} / \mathrm{ano}$.

$\mathrm{O}$ volume de efluentes que é descarregado em direção as águas subterrâneas $\left(\mathrm{q}_{\mathrm{e}}\right)$ é de $90 \%$ do volume faturado $\left(\mathrm{V}_{\mathrm{f}}\right)$, desde que, $10 \%$ é efetivamente 
Consumido. Assim sendo, $\mathrm{q}_{\mathrm{e}}=0,90 \mathrm{~V}_{\mathrm{f}}$. Onde, o volume faturado (Vf) corresponde a $60 \%$ do volume de suprimento (Vs), desde que as perdas de água na rede $\left(\mathrm{q}_{\mathrm{v}}\right)$ são de $40 \%$ do volume de suprimento (MELO et al., 2012), ou seja, $\mathrm{q}_{\mathrm{v}}=$ $0,40 \mathrm{~V}_{\text {s. }}$ Assim sendo, a equação da RU toma a seguinte forma (unidades em $\mathrm{m}^{3} / \mathrm{ano}$ ou $\mathrm{mm} / \mathrm{ano}$ ):

$\mathrm{R}_{\mathrm{U}}=0,90 \mathrm{~V}_{\mathrm{f}}+0,40 \mathrm{~V}_{\mathrm{s}}$

$\mathrm{O}$ volume de suprimento, na parte da Zona Sul de Natal e na cidade de Parnamirim, foi avaliado com base nos resultados do cadastro de poços executado na bacia do Rio Pirangi, tomando por base o arquivo de dados da CAERN e da SEMARH/RN.

\section{Avaliação da recarga pelo método da variação de carga}

Trata-se da aplicação do "Water-Table Fluctuation Method", mediante o conhecimento da variação de carga no aquífero no curso de um período considerado, referido em geral a um ano hidrológico, correspondente às reguladoras do aquífero. O método da variação de carga estabelece condições de equilíbrio, ou seja, sem a explotação das águas subterrâneas por poços, influência de recarga urbana, e, a recarga proveniente da água de irrigação.

A equação utilizada na avaliação das reservas reguladoras de um aquífero livre é a seguinte:

$\mathrm{RR}==\mathrm{A} \cdot \mathrm{dh} \cdot \mathrm{S}_{\mathrm{y}}$

(4)

Onde:

$A$ é a área de ocorrência do aquífero livre, sujeito a variação sazonal do nível d'água;

$d h$ é o pico de elevação do nível d’água atribuído a recarga; obtido pela diferença entre o pico da subida do nível de água e o ponto mais baixo da curva de recessão antecedente, extrapolada no tempo do pico (HEALY e COOK, 2002);

Sy é a porosidade específica ou rendimento específico (specific yield) do aquífero (adimensional).

A avaliação da variação de carga dh requer o monitoramento dos níveis d'água de poços, o que não foi realizado neste trabalho. Na estimativa das reservas reguladoras (RR) pelo método da variação de carga foi utilizado para dh o valor de 1,6 m obtidos em estudos regionais (IPT, 1982).

A recarga efetiva, ou seja, a recarga considerando as retiradas de água e a recarga urbana foi avaliada pela seguinte equação:

$$
\mathrm{R}=\mathrm{RR}+\mathrm{W}-\mathrm{R}_{\mathrm{U}}
$$

Onde:
$R$ é a recarga efetiva em $\mathrm{m}^{3} / \mathrm{ano}$ ou $\mathrm{mm} / \mathrm{ano}$;

$R R$ é a reserva reguladora em $\mathrm{m}^{3} / \mathrm{ano}$ ou $\mathrm{mm} / \mathrm{ano}$

$W$ é a retirada de água ou volume bombeado na bacia hidrográfica do Rio Pirangi.

$R U$ é a recarga urbana atribuída à descarga dos esgotos domésticos em direção as águas subterrâneas e às perdas de água na rede de suprimento, que corresponde a $40 \%$ do volume de suprimento.

Avaliação da recarga com aplicação da lei de Darcy ao meio poroso saturado

Em condições de equilíbrio hidrodinâmico e sem a retirada de águas subterrâneas, o fluxo subterrâneo através de uma seção vertical do aquífero é equivalente á recarga vezes a área superficial que contribui para o fluxo (SCANLON et al., 2002).

A descarga natural do fluxo subterrâneo foi estimada com a aplicação da lei de Darcy, pela equação:

$\mathrm{Q}=\mathrm{T} . \mathrm{I} . \mathrm{L}$,

Onde:

$T$ é a transmissividade $\mathrm{em}^{2} / \mathrm{s}$;

I é o gradiente hidráulico (adimensional);

$L$ é a frente de escoamento em $\mathrm{km}$.

Os mapas potenciométricos elaborados por Lucena et al (2006), Figuras 3 e 4, foram utilizados na estimativa da recarga das águas subterrâneas.

Para satisfazer as condições de um fluxo estável, as retiradas de água (W) e a influência da recarga urbana $(\mathrm{Ru})$ foram consideradas. Neste caso, a equação da recarga toma a seguinte forma:

$\mathrm{R}=\mathrm{Q}+\mathrm{W}-\mathrm{R}_{\mathrm{U}}$

Onde,

$Q$ é a descarga do fluxo subterrâneo natural em $\mathrm{m}^{3}$ /ano ou $\mathrm{mm} / \mathrm{ano}$.

\section{Avaliação da recarga pelo Método hidrológico}

A recarga das águas subterrâneas foi estimada de forma indireta com base em dados de recessão ou de fluxo de base da bacia do Rio Pirangi.

Um curso de água ou uma fonte, na ausência de precipitação, e, portanto, em regime não influenciado, se alimenta mediante as águas subterrâneas correspondentes as reservas reguladoras das camadas aqüíferas, acumuladas na bacia vertente (Figura 5) ou nas estruturas hidrogeológicas no transcurso de uma determinada recarga (CASTANY, 1976; FETTER, 2001).

A curva de recessão, parte final da curva de rebaixamento de um hidrograma de uma bacia, 
ou seja, das vazões em função do tempo, representa a lei da descarga de uma camada aquífera (figura 6), a qual pode ser expressa pela seguinte equação exponencial (CASTANY, 1976; FETTER, 2001 ):

$Q_{t}=Q_{0} \cdot e^{-\alpha t}$

Onde:

$Q_{\mathrm{t}}$ é a vazão no instante t, $\mathrm{em} \mathrm{m}^{3} / \mathrm{s}$;

$Q_{0}$ é a vazão no inicio da recessão (instante $\left.\mathrm{t}_{0}\right) \mathrm{em} \mathrm{m}^{3} / \mathrm{s}$;

$e=2,717$, a base dos logaritmos neperianos;

$\alpha$ é o coeficiente de recessão da bacia;

$t$ é o tempo transcorrido desde o começo da recessão em dias.

$\mathrm{Na}$ forma de logarítimos decimais, a equação de Maillet, pode ser escrita conforme a seguir:

$\lg \mathrm{Q}_{\mathrm{t}}=\lg \mathrm{Q}_{0}-\left({ }^{\alpha} \lg \mathrm{e}\right) \mathrm{t}$

ou

$\lg \mathrm{Q}_{\mathrm{t}}=\lg \mathrm{Q}_{0}-\left({ }^{\alpha} 0,4343\right) \mathrm{t}$
A representação gráfica desta equação caracteriza uma reta que permite obter o valor do coeficiente de recessão e, por conseguinte a expressão da equação da recessão da bacia.

A integração da vazão $Q$ no tempo t permite a obtenção da equação das (RR) no instante $\mathrm{t}_{0}$ :

$$
\mathrm{RR}=\int_{t_{0}}^{\infty} Q_{t} \cdot d t
$$

$\mathrm{Ou}$

$$
\begin{aligned}
& \mathrm{RR}=\int_{t_{0}}^{\infty} Q_{0} e^{-\alpha t} d t_{p} \text { com t em segundos. } \\
& \text { Obtendo-se: } \mathrm{RR}={ }^{\frac{Q_{0}}{\alpha}} \text { ou } \mathrm{RR}=\frac{\frac{86400 Q_{0}}{\alpha}}{\alpha}
\end{aligned}
$$

De forma similar ao apresentado para o método da variação de carga, na avaliação da recarga efetiva foi considerado as retiradas de água por bombeamentos e a recarga urbana, com a aplicação da equação (5).

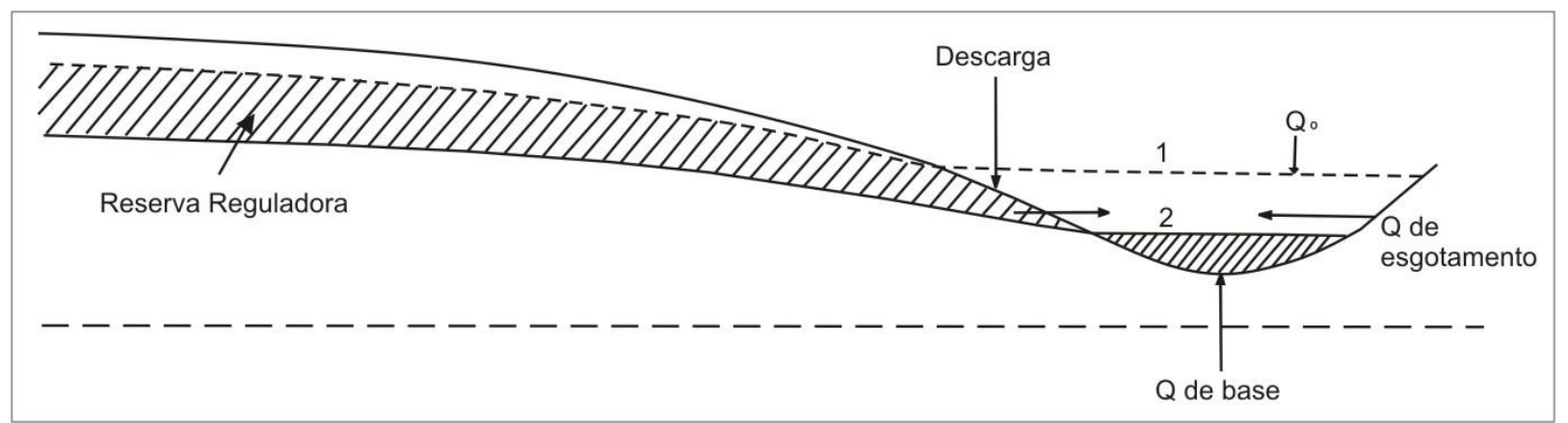

Figura 5 - Descargas das reservas reguladoras que alimentam um curso de água

Figure 5 - Discharges of the regulating reserves that feed a stream

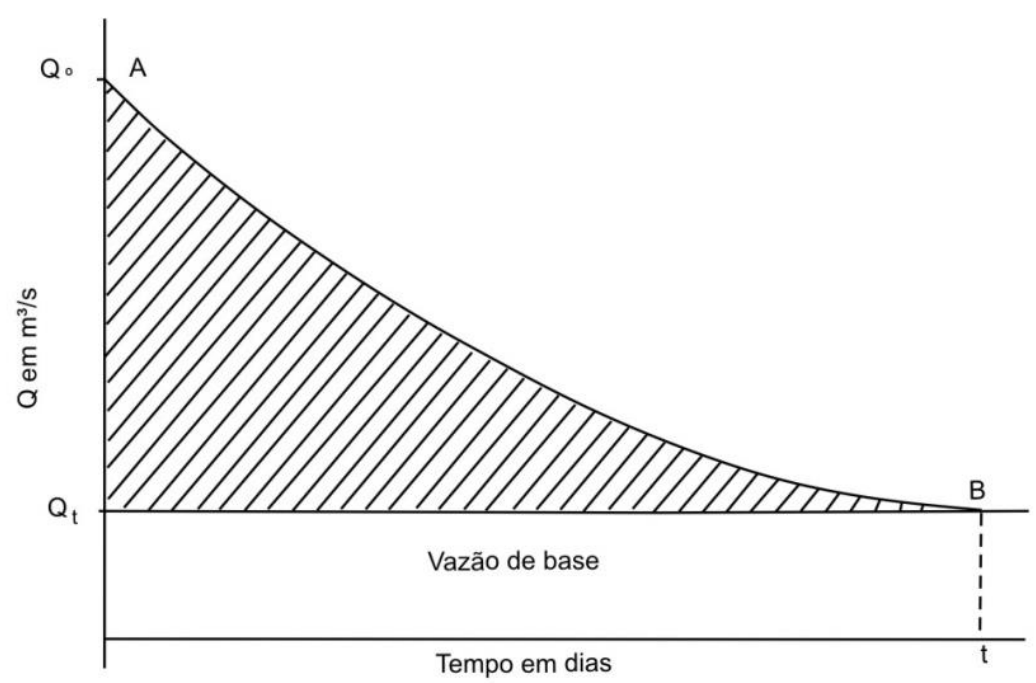

Figura 6- Curva de Recessão do hidrograma de fluxo

Figure 6 - Depletion curve of the flow hydrograph 


\section{RESULTADOS E DISCUSSÕES}

Foram cadastrados 55 poços tubulares em funcionamento penetrando o Sistema Aquífero Barreiras (SAB) na bacia hidrográfica do Rio Pirangi com produção que varia de $6,0 \mathrm{~m}^{3} / \mathrm{h}$ a $200,0 \mathrm{~m}^{3} / \mathrm{h}$, com média de $68,0 \mathrm{~m}^{3} / \mathrm{h}$. O regime de bombeamento varia de $6 \mathrm{~h} / 24$ a $24 / 24 \mathrm{~h}$. O volume total de água bombeado na bacia do Rio Pirangi foi estimado em $26 \times 10^{6} \mathrm{~m}^{3} / \mathrm{ano}$. A maioria dos poços cadastrados pertencem ao sistema público de abastecimento d'água (CAERN).

A análise dos perfis litológicos dos poços e dos resultados dos testes de bombeamento de 31 poços, totalmente penetrantes no Sistema Aquífero Barreiras, conduziu aos seguintes valores para os parâmetros hidráulicos:

A espessura do Sistema Aquífero Barreiras varia de $34,0 \mathrm{~m}$ a 95,0 $\mathrm{m}$, com média de 62,0 $\mathrm{m}$; a espessura saturada $\left(\mathrm{h}_{0}\right)$ varia de um mínimo de 19,0 m a um máximo de 69,0 m, com média de $39,0 \mathrm{~m}$; o valor mínimo da transmissividade $(\mathrm{T})$ do aquífero é de $18,8 \mathrm{~m}^{2} /$ dia ou $2,17 \times 10^{-4} \mathrm{~m}^{2} / \mathrm{s}$ e o máximo de $889,6 \mathrm{~m}^{2} / \mathrm{dia}$ ou $1,0 \times 10^{-2} \mathrm{~m}^{2} / \mathrm{s}$, com média de $383,2 \mathrm{~m}^{2} /$ dia ou $4,4 \times 10^{-3} \mathrm{~m}^{2} / \mathrm{s}$; o menor valor da condutividade hidráulica obtida foi de 0,8 $\mathrm{m} /$ dia ou $9,2 \times 10^{-6} \mathrm{~m} / \mathrm{s}$ e o máximo, de $24,5 \mathrm{~m} / \mathrm{dia}$ ou $2,8 \times 10^{-4} \mathrm{~m} / \mathrm{s}$, com média de $9,8 \mathrm{~m} / \mathrm{dia}$ ou $1,1 \times 10^{-4} \mathrm{~m} / \mathrm{s}$ (Tabela 1). A porosidade efetiva (Sy), obtida pela aplicação da equação (1), é de $16 \%$.

Para efeito de comparação e avaliação de resultados, convém informar sobre valores de porosidade específica referidos na literatura: a porosidade específica de arenitos finos varia de $2 \%$ a $40 \%$, com média de $21 \%$; no caso de arenito médio, a porosidade específica varia de $12 \%$ a $41 \%$, com média de $27 \%$; e no caso de siltitos varia de $1 \%$ a $33 \%$, com média de $12 \%$ (BATU, 1998). O Sistema Aquífero Barreiras na bacia do Rio Pirangi é constituído de arenitos finos a grossos com percentual argiloso, de forma que a taxa obtida de $16 \%$ se mostra aceitável.

A recarga urbana, avaliada na Zona Sul da cidade de Natal e na área da cidade de Parnamirim, é apresentada a seguir:

De acordo com os resultados do cadastro, o volume de suprimento (Vs) na Zona Sul da cidade de Natal é cerca de $1016 \mathrm{~m}^{3} / \mathrm{h}$ ou $8,9 \times 10^{6}$ $\mathrm{m}^{3} /$ ano e, portanto, o volume faturado é de $5,34 \times 10^{6} \mathrm{~m}^{3} /$ ano $(\mathrm{Vf}=.0,60 \mathrm{Vs}$ ); assim sendo, a descarga dos esgotos $\left(\mathrm{q}_{\mathrm{e}}\right)$ é de $4,8 \times 10^{6} \mathrm{~m}^{3} / \mathrm{ano}$ $\left(\mathrm{q}_{\mathrm{e}=0}=90 \mathrm{Vf}\right)$. As perdas de água na rede, correspondente a $40 \%$ do volume de suprimento, são de $3,56 \times 10^{6} \mathrm{~m}^{3} /$ ano $(0,40 \mathrm{Vs})$. A recarga urbana na parcela da Zona Sul de Natal, é, portanto, da or- dem de $8,36 \times 10^{6} \mathrm{~m}^{3} /$ ano. No caso da cidade de Parnamirim, o volume de suprimento $\left(\mathrm{V}_{\mathrm{s}}\right)$ é cerca de $1577 \mathrm{~m}^{3} / \mathrm{h}$ ou $13,8 \times 10^{6} \mathrm{~m}^{3} / \mathrm{ano}$, e, seguindo procedimento similar ao apresentado no caso anterior, resulta, na cidade de Parnamirim, em uma recarga urbana de $12,9 \times 10^{6} \mathrm{~m}^{3} /$ ano. A recarga urbana na bacia do Rio Pirangi, é, portanto, da ordem de $21,3 \times 10^{6} \mathrm{~m}^{3} /$ ano.

A aplicação do valor da porosidade efetiva $(\mathrm{Sy}=16 \%)$ e da variação sazonal de carga adotada ( $\mathrm{dh}=1,6 \mathrm{~m})$, na equação (4), no domínio da área efetiva de ocorrência do aquífero Barreiras $\left(A=420 \mathrm{~km}^{2}\right.$, resulta num montante das reservas reguladoras (RR) de $107,5 \times 10^{6} \mathrm{~m}^{3} . \mathrm{Na}$ avaliação da recarga (R), com a aplicação da equação (5), foram estabelecidas as condições de equilíbrio hidrodinâmico requeridas, o que resultou na recarga $(R)$ de $112,1 \times 10^{6} \mathrm{~m}^{3} /$ ano. Neste caso, foi utilizado o volume de água explotado por poços $\left(26 \times 10^{6} \mathrm{~m}^{3} / \mathrm{ano}\right)$ e o montante da recarga urbana $\left(21,4 \times 10^{6} \mathrm{~m}^{3} / \mathrm{ano}\right)$. A área efetiva de ocorrência do Sistema Aquífero Barreiras na bacia do Rio Pirangi é de $420 \mathrm{~km}^{2}$. Assim sendo, a recarga em unidade de altura de lâmina d'água é de $267 \mathrm{~mm} / \mathrm{ano}$, o que equivale a uma taxa de infiltração de $17,3 \%$, tomando por base a precipitação anual média $1539 \mathrm{~mm}$ (média da série histórica 1995-2010 (segundo informação da Empresa de Pesquisa Agropecuária do RN -EMPARN).

A aplicação da lei de Darcy ao meio poroso saturado na avaliação da recarga, conforme apresentado anteriormente, requer o conhecimento das características do fluxo, da transmissividade e da condutividade hidráulica do aquífero.

A configuração das equipotenciais nos mapas potenciométricos apresentados (figuras $3 \mathrm{e}$ 4) é perfeitamente similar, denota-se, entretanto a diferença nas cargas hidráulicas, as quais são maiores no período chuvoso e menores no período seco.

Tomando os mapas individualmente pode-se observar que as maiores cargas hidráulicas estão situadas nas zonas de tabuleiros de maior elevação topográfica e preferencialmente planas, que constituem as zonas preferenciais de recarga; enquanto que nas zonas de declividade, as cargas diminuem até atingir os valores mais baixos nos vales dos rios e riachos, que constituem os domínios de descarga das águas subterrâneas.

As linhas de fluxo, conforme mostram os mapas, seguem a orientação geral de oeste para leste em direção ao mar com inflexões para os rios e riachos principais, apresentando um comportamento típico de sistemas aquíferos livres. 
Há um aumento relativo de zonas de recarga no período chuvoso e a presença de uma zona preferencial de maior transmissividade no setor sudoeste da área (LUCENA et al., 2006).

Para avaliação da vazão do fluxo subterrâneo natural $(\mathrm{Q})$ tomou-se como referência a curva potenciométrica de $16 \mathrm{~m}$, cuja frente de escoamento é de cerca de $50 \mathrm{~km}(\mathrm{~L})$ e gradiente hidráulico médio (I) de 1\%. A transmissividade (T) média, é de $383,2 \mathrm{~m}^{2} /$ dia (tabela 1 ) ou $4,4 \times 10^{-}$ $3 \mathrm{~m}^{2} / \mathrm{s}$. Aplicando esses dados a lei de Darcy $(\mathrm{Q}=\mathrm{TIL})$, tem-se como resultado a vazão de fluxo subterrâneo natural (Q) de $2,2 \mathrm{~m}^{3} / \mathrm{s}$ ou $66,0 \times 10^{6}$ $\mathrm{m}^{3} / \mathrm{ano}$.

O método de Darcy é válido para condições de equilíbrio hidrodinâmico, de forma que as retiradas de água $\left(\mathrm{W}=26,0 \times 10^{6} \mathrm{~m}^{3} / \mathrm{ano}\right)$ e a in- fluência da recarga urbana (RU $\left.=21,3 \times 10^{6} \mathrm{~m}^{3} / \mathrm{ano}\right)$ foram consideradas, de maneira similar ao apresentado no método da variação de carga. Aplicando esses valores na equação (7), resulta uma recarga (R) de $70,7 \times 10^{6} \mathrm{~m}^{3} /$ ano ou $168 \mathrm{~mm} / \mathrm{ano}$, o que corresponde a uma taxa de infiltração de $11 \%$.

Foram efetuadas medições mensais das descargas do Rio Pirangi no seu curso mais inferior no período de 2 anos, Fevereiro 2000 a dezembro 2001 (LUCENA et al., 2006). Os resultados obtidos (Tabela 2) foram interpretados no âmbito deste trabalho tendo em vista a caracterização dos períodos de recessão (Figura 7).

As retas representativas das curvas de recessão obtidas das descargas do Rio Pirangi (anos 2000 e 2001) são apresentadas nas figuras 8 e 9.

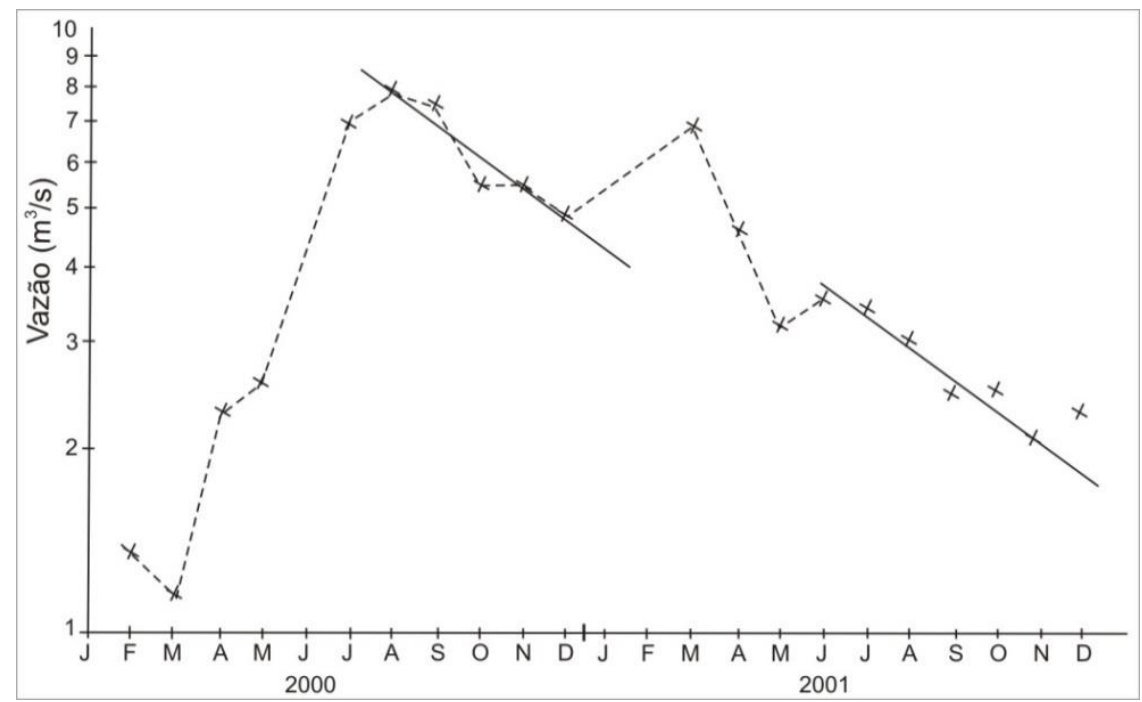

Figura 7 - Caracterização da recessão no Rio Pirangi

Figure 7 - Characterization of the recession in the Pirangi River

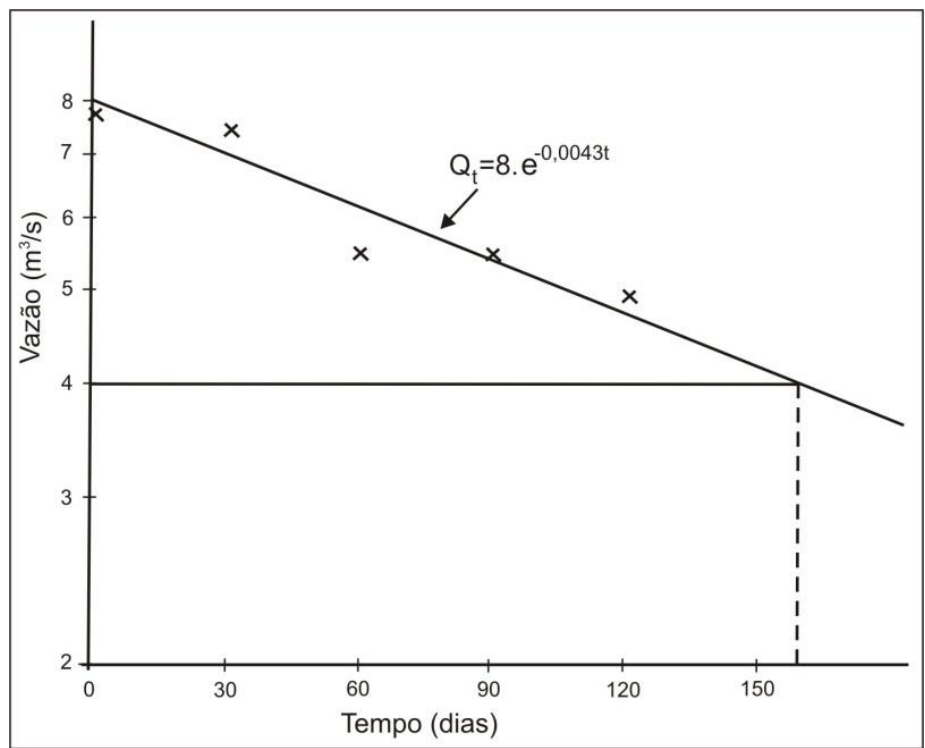

Figura 8 - Recessão na Bacia do Rio Pirangi (2000)

Figure 8 - Recession in the Pirangi River basin (2000) 


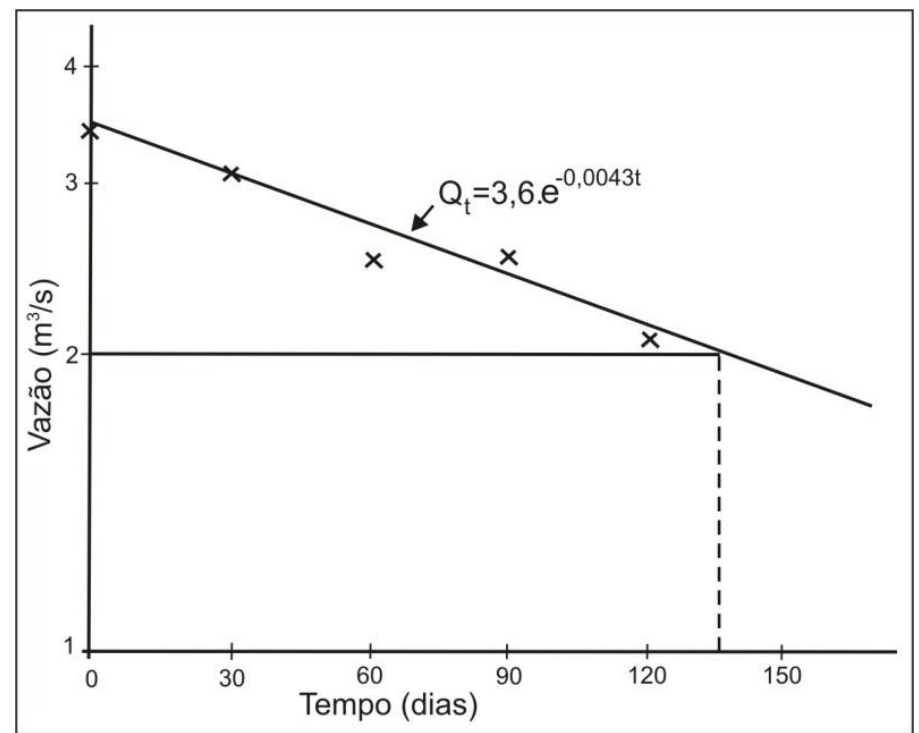

Figura 9 - Recessão na Bacia Hidrográfica do Rio Pirangi (2001)

Figure 9 - Recession in the Pirangi River basin (2001)

Observe que no ano de 2000, a descarga $\mathrm{Q}_{0}$ na origem é de $8 \mathrm{l} / \mathrm{s}$, e, para Q igual a $4 \mathrm{~m}^{3} / \mathrm{s}, \mathrm{t}$ é de 159 dias. No ano 2001, a descarga $\mathrm{Q}_{0}$ na origem é de $3,6 \mathrm{~m}^{3} / \mathrm{s}$. neste caso, tomando $\mathrm{Q}$ igual a $2 \mathrm{~m}^{3} / \mathrm{s}$, t é de 135 dias. Aplicando esses resultados na equação (8), resulta coeficientes de recessão praticamente iguais, da ordem de 0,0043 . As equações correspondentes de recessão da bacia hidrográfica do Rio Pirangi, nos dois casos apresentados, assumem a seguinte forma:

$$
\begin{aligned}
& \text { Ano 2000: } Q_{\mathrm{t}}=8 \cdot \mathrm{e}^{-0,0043 t} \\
& \text { Ano 2001: } \mathrm{Q}_{\mathrm{t}}=3,6 \mathrm{e}^{-0,0043 t}
\end{aligned}
$$

Aplicando estas equações aos respectivos casos em avaliação se obtém:

Ano 2000: $\mathrm{RR}=160 \times 10^{6} \mathrm{~m}^{3}$

Ano 2001: $\mathrm{RR}=72 \times 10^{6} \mathrm{~m}^{3}$.

Como observado, as reservas reguladoras obtidas nos dois anos são muito diferentes. Isto ocorre haja vista que no ano 2000 foi um ano de elevadas precipitações pluviométricas, que ultrapassou a média anual $(1539 \mathrm{~mm})$ em cerca de $40 \%$. Já em 2001, foi registrada uma precipitação de apenas $988 \mathrm{~mm}$ que representa aproximadamente $36 \%$ mais baixa que a média anual. Considerando que a média entre as chuvas registradas nos dois anos corresponde praticamente à média geral de $1539 \mathrm{~mm}$, acha-se conveniente tomar como representativo das reservas reguladoras a média entre os valores obtidos, ou seja, $116 \times 10^{6}$ $\mathrm{m}^{3}$. O aquífero estando sob explotação e influenciado por recarga urbana, como é o caso do Sistema Aquífero Barreiras, na verdade, a recarga efetiva corresponde ao somatório das reservas reguladoras obtidas com o volume anual de água bombeado do aquífero $\left(26 \times 10^{6} \mathrm{~m}^{3}\right)$ menos a recarga urbana $\left(21,4 \times 10^{6} \mathrm{~m}^{3}\right)$, o que resulta $120,6 \times 10^{6} \mathrm{~m}^{3}$ ou $287 \mathrm{~mm} / \mathrm{ano}$, correspondendo a uma taxa de infiltração de $18,6 \%$.

Entre os métodos utilizados e os correspondentes resultados obtidos na avaliação de recarga são feitas a seguir considerações pertinentes ao assunto.

O método da variação de carga é fácil de aplicar, porém apresenta incertezas quanto aos resultados obtidos, o que se atribui a grande variabilidade da condutividade hidráulica do aquífero, utilizada na estimativa da porosidade especifica ou rendimento especifico. Ressalta-se ainda, que a variação de carga dh foi tomada como equivalente ao valor obtido em estudos regionais.

A recarga avaliada tomando por base a lei de Darcy também apresenta incertezas devido à alta variabilidade da transmissividade (diversas ordens de magnitude). Outro fator delicado a ser considerado é o gradiente hidráulico (I), o qual foi estimado com base em mapas potenciométricos com diferentes possibilidades de caracterização do mesmo. A experiência tem mostrado que a recarga avaliada com aplicação da lei de Darcy fornece em geral valores mais baixos que os outros métodos (MELO et al., 2013).

$\mathrm{O}$ método hidrológico utiliza dados de descarga do Rio Pirangi em uma única seção do rio, no seu curso inferior, com influência sobre toda bacia, com fácil avaliação do período recessivo nos dois anos de monitoramento, o que permitiu a avaliação da recarga de forma mais sistemática e efetiva. A restrição que se faz ao método é quanto ao pequeno número de dados disponibi- 
lizado e utilizado na avaliação da recarga nos períodos de recessão e ao curto período de monitoramento.

\section{CONSIDERAÇÕES FINAIS}

A estimativa da recarga das águas subterrâneas da bacia hidrográfica do Rio Pirangi, apresentada neste artigo, são aproximadas e tão somente indicativas de ordem de grandeza. As incertezas apresentadas para cada método de avaliação se atribuem fundamentalmente à limitação de informações sobre os parâmetros hidráulicos e a complexidade do sistema hidrogeológico, o qual é representado por um sistema aquífero livre com ocorrência de semi-confinamentos localizados. Neste caso, convém ressaltar a presença de uma frequente variação lateral e vertical de fácies de arenitos para argilitos pouco ou não arenosos e variação nas espessuras saturadas do aquífero, condicionadas pela estrutura geológica do terreno.

Dentre os métodos de avaliação de recarga utilizados, o método da variação de carga ("Water-Table Fluctuation Method") é o mais fácil de aplicar. A incerteza quanto ao resultado obtido é devido, fundamentalmente, ao valor da porosidade específica utilizado, resultado de estimativa com base na média das condutividades hidráulicas obtidas neste trabalho, cujos valores apresentam uma gama de variação bastante eleva-

\section{REFERÊNCIAS}

ALVAREZ, R. H.; NIEDZIELSKI, H. Estimacion de la recarga em La subcuenca Rio de las Avenidas Pachuca Hidalgo México. In: Congresso Latino-Americano de Hidrologia . Subterrânea ,3, México, 1996. Anais do 3을 Congresso Latino-americano.

BATU, V. Aquifer Hydraulics: a comprehensive guide to hydrogeologic Data analyses. John Wiley \& Sons, Inc. Canada. 727p, 1998.

BEZERRA, F.H.R.; LUCENA, L. R. F; SAAD, A.; MOREIRA, J. A. M.; LINS, F. A. P. L.; NOGUEIRA, A. M. B.; MACEDO, J. W. P.; NAZARÉ JR. D. Estruturação Neotectônica do litoral de Natal-RN com base na correlação entre dados geológicos, geomorfológicos de gravimétricos. In SIMPOSIO NACIONAL DE ESTUDOS TECTÔNICOS, IV, Belo Horizonte. Anais. Belo Horizonte-MG. SBG, Núcleo MG. Bol. 12: p.317-321, 1993.

BORGES, A. N. Implicações ambientais na bacia hidrográfica do Rio Pitimbú, RN, decorrentes das diversas formas de uso e ocupação do solo. Dissertação de Mestrado. Programa de Pós Graduação em Engenharia Sanitária da UFRN, 2002.

CASTANY, G. Prospección y explotación de las aguas subterráneas. Barcelona: Omega,1976. da. No caso da aplicação da lei de Darcy ao meio poroso saturado, as incertezas são devido a grande variabilidade da transmissividade do aquífero, ressaltando-se ainda que este método é reconhecido por fornecer valores relativamente baixos de taxas de infiltração. O método hidrológico, com medições em uma única seção do rio, fornece informações de toda a bacia hidrográfica, enquanto que técnicas hidrogeológicas requerem um número apreciável de pontos distribuídos regularmente na área de estudo para que as informações sejam confiáveis. Neste caso, as restrições ao valor obtido no método hidrológico, são atribuídas ao pequeno número de dados de descarga do rio nos períodos das recessões observadas e ao curto período de monitoramento. A recarga a ser considerada no âmbito deste trabalho corresponde a média dos valores obtidos pelos métodos da variação de carga e da técnica hidrológica, ou seja, de $277,0 \mathrm{~mm} /$ ano que corresponde a $18,0 \%$ da precipitação anual.

Tendo em vista as incertezas apresentadas na avaliação da recarga, durante a explotação das águas subterrâneas, recomenda-se o monitoramento automatizado do nível d'água dos poços e das descargas dos rios, tendo em vista o controle da explotação e a obtenção de dados que levem à avaliação mais precisa dos recursos de águas subterrâneas.

DUARTE, C. R.; PETTA, E. A.; MEDEIROS, C. N.; ARAUJO, L. P. Mapeamento do uso e ocupação do solo da Bacia do rio Pitimbú, RN. Anais II Simpósio Regional de Geoprocessamento e Sensoriamento Remoto, Aracaju, SE, 2004.

FETTER, C.W. Applied hydrogeology. $4^{\text {th }}$ ed. Prentice-Hall, Inc. Upper Saddle River, New Jersey 07458. 598p, 2001.

FOSTER, S. S. D; MORRIS, B.; LAWRENCE, A.; CHILTON, JOHN. Groundwater impacts and issues in developing cities - An Introductory review. In: Groundwater in the Urban Environment. Selected City Profiles. Volume 21 of the International Contributions to Hydrogeology. Rotterdam: A.A. Bakema, 1999.

HEALY, R. W.; Cook, P. G. Using groundwater levels to estimate recharge. Hydrogeology Journal, v. 10, n.1, p. 91-109, 2002.

IPT. Estudo Hidrogeológico Detalhado do Estado do Rio Grande do Norte. Instituto de Pesquisas Tecnológicas do Estado de São Paulo (IPT)/Secretaria de Planejamento do Estado do Rio Grande do Norte. Natal, 9 Vol, 1982. 
KOBAYASHI, R. T.; MOREIRA, L. F. P.; ANDRADE, H. T. A.. Pitimbu River low land portion water and sediment monitoring data, Natal, Brazil. International Congress an Environment Moddeling and software, Ottawa, Canada, 2010.

LUCENA, L.R.F.; QUEIROZ, M. A. Considerações sobre as influências de uma tectônica cenozoica na pesquisa e prospecção de recursos hídricos - o exemplo do litoral sul de Natal- RN, Brasil. Revista Águas Subterrâneas, ABAS. V. 1, nº 15, p. 81-88, 1996.

LUCENA, R. F. L.; ROSA FILHO, E. F.; BITTENCOURT, A. V. L. A potenciometria do aquífero Barreiras no setor oriental da bacia do Rio Pirangi-RN e considerações sobre a relação com os mananciais superficiais. Revista Águas Subterrâneas, ABAS. V. 18, 2004.

LUCENA, R. F. L.; BECEGATO, V. A.; CABRAL, J. B. $P$. Considerações sobre a potenciometria do aquífero Barreiras e a influência da estruturação regional nesse contexto - setor da bacia do rio Pirangi-RN. Revista Eletrônica do Curso de Geografia do Campus Jatai - UFG. No 7, 2006.

MELO, J.G.; DE MORAIS, S. D.; ALVES, R. S.; VAS-
CONCELOS, M. B.. Avaliação dos recursos hídricos do Aquífero Barreiras na bacia do Rio Maxaranguape, RN. Revista Agua Subterrânea, ABAS. V 27, n 1, p. 5364, 2013.

MELO, J.G.; QUEIROZ, M. A.. The effects of urban depelopment on the groundwater recharge and its quality in Natal, RN, Brasil. In: SEILER, K. P.; WOHNLICH, S.. New Approches Caracterizing Groundwater Flow. Netherland: v. 2, p. $1233-1236$, 2001

MELO, J.G.; VASCONCELOS, M. B.; DE MORAIS, S. D.; ALVES, R. S.. Avaliação hidrogeológica da Zona Norte da cidade de Natal, RN, e os problemas associados ao desenvolvimento urbano. Revista Brasileira de Recursos Hídricos, RBRH. V. 17, no 1, p. 123-134, 2012.

PAZDRO, Z. Hydrogeologia Ogólna, W.G. Warszawa, p.575, 1983.

SCANLON, B. R.; HEALY, R. W.; COOK, P. G. Choosing appropriate techniques for quantifying groundwater recharge. Hydrogeology Journal, V. 10, n. 1, p. 1839, 2002. 